\title{
Corps étrangers oculaires : évolution des rôles professionnels et des coûts pour le régime de santé public du Québec
}

\author{
Benoît Tousignant, \\ O.D., M.Sc., M.S.P., FAAO \\ École d'optométrie, \\ Université de Montréal, \\ École de santé publique, \\ Université de Montréal \\ Drissa Moriba Coulibaly, \\ B.Optom, M.Sc. \\ Institut d'ophtalmologie \\ tropicale d'Afrique (IOTA) \\ Julie Brûlé, O.D., M.Sc., FAAO \\ École d'optométrie, \\ Université de Montréal \\ Jacques Gresset, \\ O.D., Ph.D., FAAO \\ École d'optométrie, \\ Université de Montréal
}

\begin{abstract}
RÉSUMÉ
OBJECTIF

En 2003, les optométristes du Québec ont été autorisés en vertu de la loi à extraire des corps étrangers oculaires superficiels, une partie de ce service étant couverte par le régime universel d'assurance-maladie. Cette étude analyse l'évolution du rôle des professionnels qui prennent en charge ce problème (optométristes, ophtalmologistes, urgentologues et omnipraticiens) et les coûts connexes des soins de santé publics.
\end{abstract}

\section{MÉTHODES}

Des données tirées du régime provincial d'assurance-maladie ont été combinées aux statistiques démographiques et annuelles concernant la maind'œuvre du secteur de la santé. Dans l'ensemble des professions et des régions sociosanitaires, la variation des taux annuels des problèmes traités a été calculée, de même que la variation des coûts des soins de santé publics. Les pentes de régression linéaire de ces variations ont été utilisées comme indicateurs des tendances linéaires.

\section{RÉSULTATS}

Entre 2010 et 2016, la proportion de cas traités par les optométristes a augmenté considérablement, passant de $32 \%$ à $44 \%(p<0,007)$. Chez les omnipraticiens, la proportion de cas traités a diminué considérablement, passant de $49 \%$ à $33 \%(p<0,0001)$. L'augmentation du nombre de cas pris en charge se constate dans presque toutes les régions sociosanitaires, la hausse atteignant $19 \%$. Les coûts des soins de santé ont augmenté considérablement dans le cas des optométristes $(p<0,008)$ et des ophtalmologistes $(p<0,004)$ et diminué considérablement par rapport aux omnipraticiens ( $p<0,001)$. En 2016, les optométristes ont pris en charge $44 \%$ des cas, ce qui représente $13 \%$ des coûts des soins de santé connexes.

\section{CONCLUSION}

Au Québec, les optométristes prennent dorénavant en charge la plus grande partie des corps étrangers oculaires superficiels. Cela est contrebalancé par une diminution apparente de la proportion de cas pris en charge par les omnipraticiens.

MOTS-CLÉS :

corps étrangers oculaires; optométrie; ophtalmologie; traitement d'urgence; accessibilité des services de santé; dépenses en santé

\section{INTRODUCTION}

Les corps étrangers oculaires superficiels sont de petits corps solides qui se projettent dans un œil, endommageant les couches antérieures de la cornée ou contribuant à la conjonctivite. Ils touchent le plus souvent les hommes et découlent de blessures liées au travail ${ }^{1-3}$. Bien que les corps étrangers oculaires superficiels présentent moins de risques de morbidité que les corps étrangers intraoculaires, ils présentent un risque d'incapacité visuelle et entraînent souvent des coûts découlant de la rémunération du personnel ${ }^{4-6}$. 
La prise en charge des corps étrangers oculaires superficiels est un acte de soin oculovisuel de première ligne qui consiste, pour le professionnel formé, à localiser et à retirer le corps étranger sous anesthésie topique locale avec des instruments ophtalmiques appropriés, habituellement avec une lampe à fente ${ }^{7}$.

Au Québec, province canadienne, le coût de l'extraction d'un corps étranger oculaire superficiel par un ophtalmologiste, urgentologue ou médecin de famille a été couvert jusqu'au 2003 par le programme universel de soins de santé publics (Régie de l'assurance-maladie du Québec, RAMQ).

Les ophtalmologistes sont des médecins spécialisés qui fournissent des soins oculovisuels médicaux et chirurgicaux, ce qui comprend la prise en charge des corps étrangers oculaires superficiels. En 2016, on comptait 400 ophtalmologistes au Québec (1 pour 20815 habitants)9. Bien que leurs services soient de plus en plus recherchés, leur nombre augmente à un rythme plus lent que celui de la population vieillissante qui a besoin de services ophtalmiques ${ }^{10,11}$. Les urgentologues sont des médecins spécialisés qui prodiguent des soins dans les salles d'urgence des hôpitaux, ce qui comprend la prise en charge des corps étrangers oculaires superficiels et le renvoi du patient chez un ophtalmologiste au besoin. On dénombre 200 urgentologues au Québec (1 pour 41631 habitants) ${ }^{9}$. En 2016, il y avait 10779 omnipraticiens au Québec (1 pour 772 habitants) et ils représentaient plus de $85 \%$ des médecins travaillant en salle d'urgence (Fédération des médecins omnipraticiens du Québec [FMOQ], communication personnelle, 2017). C'est donc dire que les omnipraticiens prennent aussi en charge les corps étrangers oculaires superficiels. Les délais dans les salles d'urgence des hôpitaux peuvent atteindre 48 heures $^{12}$.

Au Québec, les optométristes sont les principaux fournisseurs de soins oculovisuels; ils sont formés pour identifier et traiter certains types de pathologies oculaires par des médicaments topiques ou oraux ${ }^{13}$. La majorité des optométristes travaillent en cabinet privé, mais ils peuvent facturer la RAMQ pour la prestation de services à des groupes particuliers (enfants, personnes âgées) ou à tous les groupes d'âge dans certains cas (urgences oculaires, soins du diabète, aide sociale). Les 1499 optométristes (1 pour 5554 habitants) répartis dans toutes les régions sociosanitaires de la province offrent à la population un accès à des soins oculovisuels ${ }^{14,15}$. En 2003, à la suite d'un élargissement de leur champ d'exercice, les optométristes ont été autorisés en vertu de la loi à prendre en charge les corps étrangers oculaires superficiels qui ne menacent pas l'axe visuel central ${ }^{13}$. La RAMQ couvre la partie diagnostique (examen) des honoraires professionnels connexes et la partie thérapeutique (extraction) est payée par le patient ou son assurance-maladie complémentaire privée.

À ce jour, aucune étude n'a évalué l'impact de la participation des optométristes à la prise en charge des corps étrangers oculaires superficiels au Québec depuis que leur champ d'exercice a été élargi. L’objectif de cette étude est d'analyser l'évolution et la répartition de la prise en charge des corps étrangers oculaires superficiels par les différents professionnels concernés ainsi que l'évolution des coûts liés à la RAMQ.

\section{MATÉRIEL ET MÉTHODES}

Les données sur la prise en charge des corps étrangers oculaires superficiels (nombre d'actes, nombre de patients, nombre de professionnels assurant la prestation de services et coûts) par les optométristes, les ophtalmologistes, les urgentologues et les omnipraticiens ont été obtenues de la RAMQ. Entre 2003 et 2009, les données de la RAMQ sur les optométristes qui prennent en charge les corps étrangers oculaires superficiels n'étaient pas disponibles car le codage de ce type de problème a été effectué avec un code d'urgence oculaire non déterminé. En 2009, un sous-code particulier pour les optométristes a été instauré concernant les corps étrangers oculaires superficiels. Par conséquent, l'étude porte sur la période de 2010 à 2016. On a obtenu les codes de procédure et de diagnostic pertinents pour toutes les professions après avoir consulté les manuels de facturation de la RAMQ, le personnel de facturation de la RAMQ dans les hôpitaux, le Collège des médecins du Québec et l'Association des optométristes du Québec (tableau 1).

Ces données ont été combinées à la répartition des quatre types de professionnels selon les régions sociosanitaires (RSS), ainsi qu'aux données démographiques gouvernementales de l'Institut de la statistique du Québec ${ }^{16,17}$.

Les proportions absolues de ces professions prenant en charge les corps étrangers oculaires superficiels dans l'ensemble des RSS ont été établies pour 2010 et 2016 de même que leurs variations respectives. Les taux relatifs de patients traités pour des corps étrangers oculaires superficiels par 100000 habitants (CÉOS/100 000) ont également été établis pour chaque profession. Un « patient » est défini comme une personne qui a consulté au moins une fois pour un problème de santé facturé à la RAMQ par année de référence. Les coûts totaux facturés à la RAMQ 
pour les corps étrangers oculaires superficiels par 100000 habitants (CÉOS\$/100 000) ont été établis pour chaque profession. Une régression linéaire simple a servi à analyser la variation des CÉOS/100 000 et des CÉOS\$/100 000 prévue pour les années de l'étude et pour chacune des professions. Des tests t sur les pentes de régression ont servi à évaluer l'importance des tendances linéaires au cours de la période de l'étude.

Tableau 1 : Codes de procédure et de diagnostic relatifs à la prise en charge des corps étrangers oculaires superficiels au Québec, 2010-2016

\begin{tabular}{|l|c|c|}
\hline & Codes de procédure & Codes de diagnostic \\
\hline Omnipraticiens & 07204,07154 & $9309,9300,9301$ \\
\hline Ophtalmologistes & 07204,07159 & $9309,9300,9301$ \\
\hline Urgentologues & 07159 & $9309,9300,9301$ \\
\hline Optométristes & 9019, et sous-code A00.8* & S.0. \\
\hline
\end{tabular}

* Le sous-code A00.8 n'était pas disponible entre 2003 et 2009 car les corps étrangers oculaires superficiels (CÉOS) pris en charge par les optométristes correspondaient à un code d'urgence oculaire non spécifique. En 2009, ce sous-code particulier aux optométristes a été instauré pour désigner les corps étrangers oculaires superficiels.

Des analyses statistiques ont été effectuées à l'aide de SPSS (IBM, 2016. IBM SPSS Statistics pour Windows, version 24.0. Armonk, NY).

\section{RÉSULTATS}

Gestion des corps étrangers oculaires superficiels par profession

Entre 2010 et 2016, pour l'ensemble de la province et pour toutes les professions combinées, le taux de CÉOS/100 000 a diminué et ce changement est significatif sur le plan statistique. La proportion de corps étrangers oculaires superficiels pris en charge par les optométristes est passée de $32 \%$ à $44 \%$ (figure 1). Le tableau 2 montre le modèle de régression $\left(\mathrm{r}^{2}=0,79, \mathrm{~F}[1,6]=19,3, \mathrm{t}=4,4, p=0,007\right)$; il illustre une tendance à la hausse significative occasionnée par une augmentation annuelle de 6,9 CÉOS/100 000.

Figure 1 : Variation de la prise en charge des corps étrangers oculaires superficiels par les professionnels du Québec (2010 à 2016)
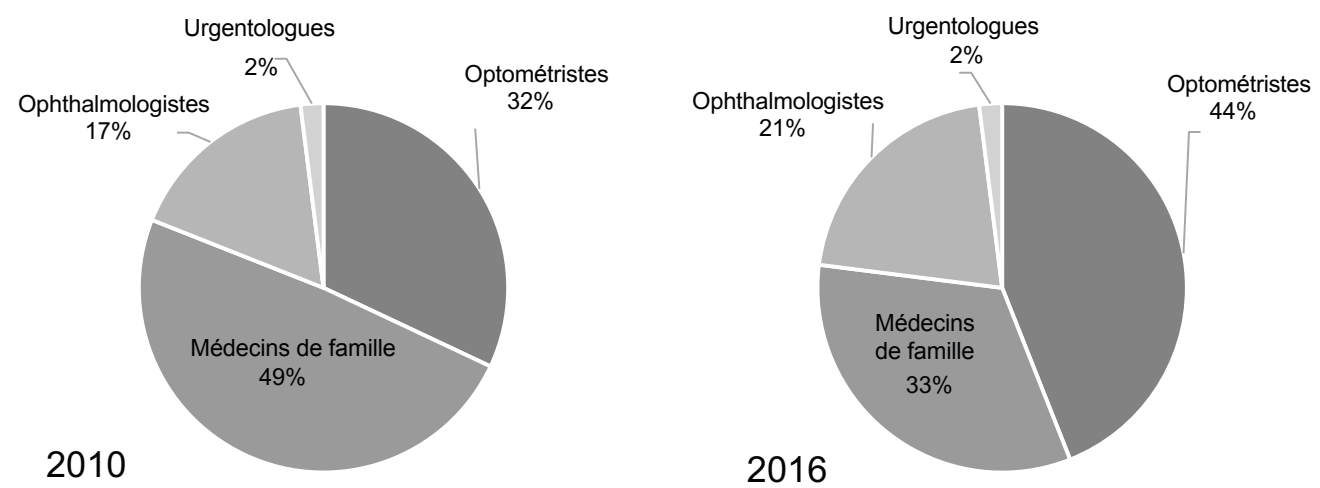
Tableau 2 : Analyse de régression linéaire de la variation annuelle du taux de corps étrangers oculaires superficiels (pour 100000 personnes) pour diverses professions de la santé, Québec, Canada, 2010-2016

\begin{tabular}{|l|c|c|c|c|c|c|c|}
\hline & $\mathbf{r}^{2}$ & $\mathbf{F}$ & $\mathbf{d f}$ & $\mathbf{t}$ & $p^{*}$ & $\begin{array}{c}\beta \mathbf{0} \\
\text { (pente) }\end{array}$ & $\beta \mathbf{1}$ (ordonnée) \\
\hline Toutes les professions & 0,89 & 41,6 & 1,6 & $-6,4$ & 0,001 & $-7,7$ & 15943,0 \\
\hline Optométristes & 0,79 & 19,3 & 1,6 & 4,4 & 0,007 & 6,9 & $-13703,7$ \\
\hline Omnipraticiens & 0,96 & 121,0 & 1,6 & $-11,0$ & $<0,001$ & $-17,7$ & 35752,95 \\
\hline Ophtalmologistes & 0,57 & 6,6 & 1,6 & 2,6 & 0,05 & 3,1 & $-6185,9$ \\
\hline Urgentologues & 0,02 & 0,1 & 1,6 & $-0,28$ & 0,8 & $-0,04$ & 79,9 \\
\hline
\end{tabular}

* Les valeurs de $p<0,05$ indiquent une pente de régression non nulle et une tendance linéaire statistiquement significative

Chez les omnipraticiens, la proportion des corps étrangers oculaires superficiels pris en charge a diminué, passant de $49 \%$ à $33 \%$ (figure 1). Le modèle de régression $\left(\mathrm{r}^{2}=0,96, \mathrm{~F}[1,6]=121,0, \mathrm{t}=-11,0, p<0,001\right)$ montre une tendance linéaire à la baisse significative en raison d'une diminution annuelle de 17,7 CÉOS/100 000. Chez les ophtalmologistes, la proportion de corps étrangers oculaires superficiels pris en charge est passée de $17 \%$ à $21 \%$ (figure 1 ). Le modèle de régression $\left(\mathrm{r}^{2}=0,57, \mathrm{~F}[1,6]=6,6, \mathrm{t}=2,6, p=0,05\right)$ montre une tendance à la hausse significative en raison d'une augmentation annuelle de 3,1 CÉOS/100 000. Chez les urgentologues, la proportion de corps étrangers oculaires superficiels pris en charge n’a pas varié de façon significative et est demeurée stable à $2 \%$; le modèle de régression n'a pas montré de tendance significative.

Variation de la prise en charge des corps étrangers oculaires superficiels dans diverses régions sociosanitaires

Au cours de la période couverte par cette étude, une augmentation de la proportion de corps étrangers oculaires superficiels pris en charge par les optométristes est observable dans toutes les RSS sauf une (tableau 3). Toutefois, cette augmentation diffère entre les régions urbaines, périurbaines et rurales. À Montréal, la proportion des corps étrangers oculaires superficiels pris en charge par des optométristes a augmenté de $12 \%$ (passant de $22 \%$ à $34 \%$ ). L'augmentation chez les optométristes a atteint $18 \%$ (passant de $41 \%$ à $59 \%$ ) dans la région périurbaine de la Montérégie mais seulement $3 \%$ (passant de $55 \%$ à $58 \%$ ) dans la région rurale des Laurentides. À l'inverse, la proportion de corps étrangers oculaires superficiels pris en charge par les omnipraticiens a diminué dans la plupart des RSS, affichant une baisse variant entre $6 \%$ et $20 \%$ (tableau 3 ).

Coûts de la prise en charge des corps étrangers oculaires superficiels payés par la RAMQ

Entre 2010 et 2016, pour l'ensemble de la province et pour toutes les professions combinées, le taux de CÉOS\$/100 000 est demeuré stable et la régression n'a montré aucune tendance linéaire significative (tableau 4). En 2010, 11 \% des coûts de la RAMQ associés aux corps étrangers oculaires superficiels ont été facturés par les optométristes; ce pourcentage est passé à $13 \%$ en 2016 (figure 2). Le modèle de régression $\left.\left(\mathrm{r}^{2}=0,79, \mathrm{~F}[1,6]=[18,2]\right), \mathrm{t}=4,3, p=0,008\right)$ montre une tendance à la hausse significative (tableau 4). En 2010, les omnipraticiens ont généré $47 \%$ des coûts de la RAMQ associés aux corps étrangers oculaires superficiels et ce pourcentage est passé à $37 \%$ en 2016 (figure 2). Le modèle de régression ( $r^{2}=0,91$, $\mathrm{F}[1,6]=51,1, \mathrm{t}=-7,2, p<0,001$ ) montre une tendance à la baisse significative (tableau 4). En 2010, les ophtalmologistes ont généré $40 \%$ des coûts de la RAMQ associés aux corps étrangers oculaires superficiels, lesquels ont grimpé à $47 \%$ en 2016 (figure 2). Le modèle de régression $\left(\mathrm{r}^{2}=0,83, \mathrm{~F}[1,6]=24,7, \mathrm{t}=4,9, p=0,004\right)$ montre une tendance linéaire à la hausse significative (tableau 4). En ce qui concerne les urgentologues, la régression ne montre aucune tendance significative.

\section{DISCUSSION}

Cette étude a examiné les variations dans la prise en charge des corps étrangers oculaires superficiels au Québec entre 2010 et 2016, après que les optométristes se soient ajoutés aux divers autres professionnels autorisés par la loi à effectuer l'extraction des corps étrangers oculaires superficiels. Au cours de la période couverte par cette étude, le nombre total de corps étrangers oculaires superficiels pris en charge par l'ensemble des professionnels au Québec a diminué. Bien que les causes soient probablement multifactorielles, la diminution peut être attribuable aux efforts déployés par le gouvernement pour améliorer la sécurité au travail grâce aux stratégies de promotion de la santé et de protection des travailleurs ${ }^{18}$. 
Tableau 3 : Variations géographiques du traitement des corps étrangers oculaires superficiels par les professionnels du Québec, 2010 à 2016.

\begin{tabular}{|c|c|c|c|c|c|}
\hline \multirow{2}{*}{$\begin{array}{l}\text { Régions } \\
\text { sociosanitaires }\end{array}$} & \multirow{2}{*}{$\begin{array}{l}\text { Type de } \\
\text { région }\end{array}$} & \multicolumn{4}{|c|}{$\begin{array}{l}\text { Corps étrangers oculaires superficiels traités par des professionnels en } 2016 \\
\qquad(\%, \text { variation par rapport à 2010) }\end{array}$} \\
\hline & & Optométristes & Omnipraticiens & Ophtalmologistes & Urgentologues \\
\hline Montréal & Urbaine & $34 \%$ & $20 \%$ & $46 \%$ & $2 \%$ (stable) \\
\hline Capitale-Nationale & Urbaine & $23 \%(+10 \%)$ & $54 \%(-8 \%)$ & $17 \%(-1 \%)$ & $6 \%(-1 \%)$ \\
\hline Laval & Périurbaine & $45 \%$ & $19 \%$ & $36 \%$ & $8 \%(+3 \%)$ \\
\hline Montérégie & Périurbaine & $58 \%$ & $21 \%$ & $21 \%$ (stable) & $0 \%(0 \%)$ \\
\hline Bas-Saint-Laurent & Rurale & $26 \%(+4 \%)$ & $49 \%(-20 \%)$ & $12 \%(+3 \%)$ & $0 \%(0 \%)$ \\
\hline $\begin{array}{l}\text { Saguenay-Lac-Saint- } \\
\text { Jean }\end{array}$ & Rurale & $52 \%(+9 \%)$ & $36 \%(-14 \%)$ & $11 \%(+4 \%)$ & $0 \%(0 \%)$ \\
\hline Mauricie & Rurale & $45 \%(+15 \%)$ & $43 \%(-14 \%)$ & $12 \%(-1 \%)$ & $0 \%(0 \%)$ \\
\hline Estrie & Rurale & $36 \%(+13 \%)$ & $52 \%(-13 \%)$ & $12 \%(+1 \%)$ & $0 \%(-1 \%)$ \\
\hline Outaouais & Rurale & $61 \%(13 \%)$ & $25 \%(-14 \%)$ & $14 \%(+1 \%)$ & $0 \%(0 \%)$ \\
\hline $\begin{array}{l}\text { Abitibi- } \\
\text { Témiscamingue }\end{array}$ & Rurale & $49 \%(+1 \%)$ & $47 \%$ (stable) & $4 \%(-1 \%)$ & $0 \%(0 \%)$ \\
\hline Côte-Nord & Rurale & $27 \%(+6 \%)$ & $57 \%(+3 \%)$ & $16 \%(-9 \%)$ & $0 \%(0 \%)$ \\
\hline $\begin{array}{l}\text { Gaspésie-Îles-de-la- } \\
\text { Madeleine }\end{array}$ & Rurale & $46 \%(+2 \%)$ & $45 \%(-6 \%)$ & $9 \%(+4 \%)$ & $0 \%(0 \%)$ \\
\hline Chaudière-Appalaches & Rurale & $28 \%(+10 \%)$ & $52 \%(-16 \%)$ & $28 \%(+10 \%)$ & $0 \%(0 \%)$ \\
\hline Lanaudière & Rurale & $46 \%(-4 \%)$ & $45 \%(+13 \%)$ & $9 \%(-9 \%)$ & $0 \%(-1 \%)$ \\
\hline Laurentides & Rurale & $58 \%(+3 \%)$ & $16 \%(-17 \%)$ & $26 \%(+14 \%)$ & $0 \%(0 \%)$ \\
\hline TOTAL & & $44 \%(+12 \%)$ & $33 \%(-16 \%)$ & $21 \%(+4 \%)$ & $2 \%(0 \%)$ \\
\hline
\end{tabular}

CÉOS : corps étrangers oculaires superficiels

Tableau 4 : Analyse de régression linéaire de la variation annuelle des coûts des soins de santé publics de la prise en charge des corps étrangers oculaires superficiels (pour 100000 habitants) pour diverses professions de la santé au Québec, Canada, de 2010 à 2016

\begin{tabular}{|l|c|c|c|c|c|c|c|}
\hline & $\mathbf{r}^{2}$ & $\mathbf{F}$ & df & $\mathbf{t}$ & $p^{*}$ & $\begin{array}{c}\beta \mathbf{0} \\
\text { (pente) }\end{array}$ & $\begin{array}{c}\beta \mathbf{1} \\
\text { (ordonnée) }\end{array}$ \\
\hline Toutes les professions & 0,32 & 2,3 & 1,6 & 1,5 & 0,2 & 149,7 & -279026 \\
\hline Optométristes & 0,79 & 18,2 & 1,6 & 4,3 & 0,008 & -159300 & 80,5 \\
\hline Omnipraticiens & 0,91 & 51,1 & 1,6 & $-7,2$ & 0,001 & 756323 & $-371,1$ \\
\hline Ophtalmologistes & 0,83 & 24,7 & 1,6 & 4,9 & 0,004 & -753512 & 379,1 \\
\hline Urgentologues & 0,74 & 14,5 & 1,6 & 3,8 & 0,01 & -122536 & 61,2 \\
\hline
\end{tabular}

* Les valeurs de $p<0,05$ indiquent une pente de régression non nulle et une tendance linéaire statistiquement significative 
Figure 2 : Variations des coûts des soins de santé publics liés à la prise en charge des corps étrangers oculaires superficiels par les professionnels du Québec (2010 à 2016)
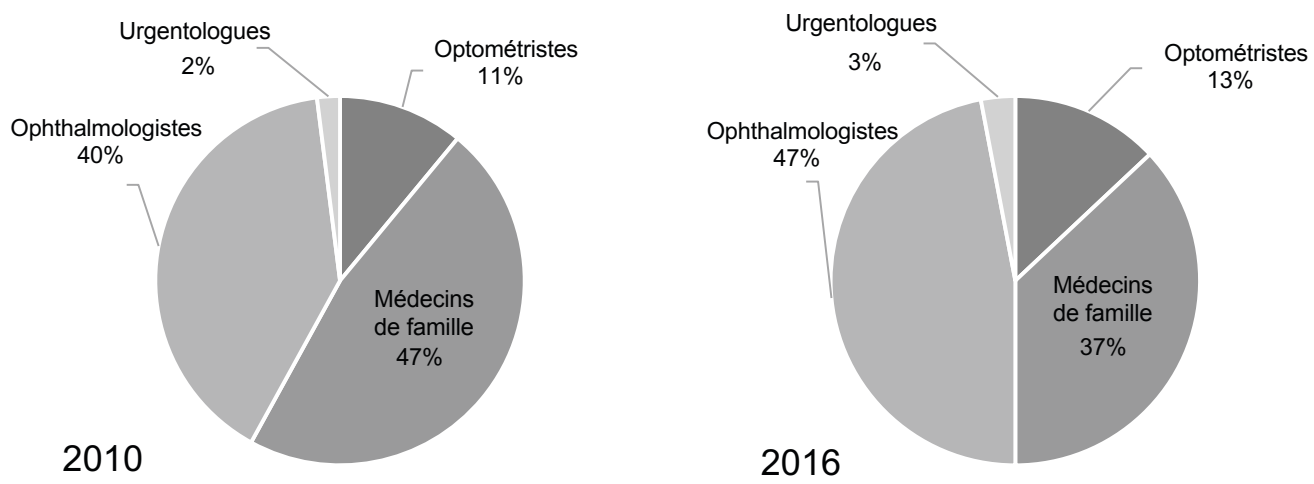

Malheureusement, aucune donnée n'est disponible pour 2003-2009, période au cours de laquelle les optométristes ont commencé à prendre en charge les corps étrangers oculaires superficiels. Toutefois, on peut supposer qu'au cours de cette période, les optométristes ont adopté de façon marquée la prise en charge des corps étrangers oculaires superficiels du fait qu'en 2010, 32 \% des extractions ont été effectuées par des optométristes (tableau 2).

Entre 2010 et 2016, à l'échelle provinciale, le nombre de CÉOS/100 000 pris en charge par les optométristes a continué d'augmenter légèrement, cette tendance linéaire étant significative et se produisant à un rythme supérieur à la diminution globale du nombre de prises en charge de corps étrangers oculaires superficiels. La proportion de corps étrangers oculaires superficiels pris en charge par les optométristes a augmenté pour atteindre $44 \%$ en 2016 , ce groupe devenant ainsi le plus important en nombre parmi les professionnels recensés (figure 1). À l'inverse, au cours de la même période, la proportion de CÉOS/100 000 pris en charge par les omnipraticiens a considérablement diminué, celle-ci passant de $49 \%$ (2010) à $33 \%$ (2016). Cette tendance a été observée en dépit de l'augmentation de $8 \%$ du nombre de omnipraticiens au cours de cette période..$^{19}$ La RSS de Lanaudière fait exception, celle-ci affichant une augmentation de $13 \%$ du nombre de corps étrangers oculaires superficiels pris en charge par les omnipraticiens parallèlement à une diminution de $4 \%$ chez les optométristes et de $9 \%$ chez les ophtalmologistes. Cette hausse est peut-être attribuable à une augmentation de $16 \%$ du nombre de omnipraticiens, soit le double de la variation à l'échelle de la province pour cette période ${ }^{19}$.

Au cours de la période couverte par cette étude, la proportion de CÉOS/100 000 n’a augmenté que légèrement chez les ophtalmologistes et est demeurée stable chez les urgentologues (figure 1). Nous présumons ainsi que la proportion accrue de prises en charge des corps étrangers oculaires superficiels par les optométristes provient en grande partie de celles auparavant attribuées aux omnipraticiens. Cette variation peut s'expliquer par un changement de comportement de la population qui souhaite obtenir des soins oculovisuels. Certains patients pourraient vouloir consulter un optométriste plutôt qu'un autre professionnel en raison de la plus grande facilité d'accès, du court délai d'obtention d'un rendez-vous et de la facilité d'obtenir un traitement, comparativement aux professionnels des salles d'urgence et aux ophtalmologistes ${ }^{20}$.

De plus, comme la plupart des extractions de corps étrangers oculaires superficiels effectuées par les omnipraticiens ont lieu dans les salles d'urgence des hôpitaux ou dans les cliniques d'urgence sans rendez-vous (FMOQ, communication personnelle, 2017), la prise en charge de ces actes par les optométristes pourrait réduire la congestion dans les services d'urgence offerts à la population.

Sur le plan géographique, l'augmentation du nombre de prises en charge de corps étrangers oculaires superficiels par les optométristes est considérable dans toute la province. Dans la plupart des RSS, la hausse atteint de 2 à $19 \%$ entre 2006 et 2010 (tableau 2). La proportion d'optométristes qui prennent en charge les corps étrangers oculaires superficiels est plus élevée dans les régions rurales et périurbaines que dans les régions urbaines. En 2016, dans certaines régions rurales, $60 \%$ des corps étrangers oculaires superficiels ont été pris en charge par des optométristes, probablement là où ces professionnels sont plus facilement accessibles. À l'inverse, en 2016, dans les RSS urbaines, 
entre $23 \%$ et $34 \%$ des corps étrangers oculaires superficiels ont été pris en charge par des optométristes, ensuite par des ophtalmologistes (Montréal) et, enfin, par des omnipraticiens (Québec). Certaines variations dans la proportion de corps étrangers oculaires superficiels pris en charge par les optométristes en milieu rural sont plutôt minimes (hausse de $2 \%$ dans la région Gaspésie-Îles-de-la-Madeleine et de $3 \%$ dans les Laurentides). Néanmoins, compte tenu du fait qu'en 2010 l'optométrie était déjà la profession la plus en vue pour prendre en charge les corps étrangers oculaires superficiels dans ces RSS (44\% dans la région Gaspésie-Îles-de-la-Madeleine et $56 \%$ dans les Laurentides), celles-ci ont rapidement opté pour cette voie parce qu'elles avaient peut-être déjà atteint une certaine stabilité entre 2003 et 2009 , soit avant la période couverte par cette étude.

Pour toutes les professions, le coût des soins de santé publics couverts par la RAMQ pour la prise en charge des corps étrangers oculaires superficiels n'a pas varié de façon significative au cours de la période couverte par cette étude (tableau 2). Toutefois, si l'on tient uniquement compte des omnipraticiens, les coûts ont diminué considérablement, ce qui correspond à leur part réduite de prise en charge des corps étrangers oculaires superficiels. Pour les optométristes, ces coûts ont augmenté légèrement (passant de $11 \%$ à $13 \%$ des coûts totaux liés à la RAMQ), à la suite de l'augmentation de leur part de prise en charge des corps étrangers oculaires superficiels au cours de la même période. Les frais de remboursement des optométristes par la RAMQ sont demeurés stables pendant cette période ${ }^{21}$. En ce qui concerne les ophtalmologistes, les coûts de la prise en charge des corps étrangers oculaires superficiels facturés à la RAMQ sont passés de $40 \%$ à $47 \%$ des coûts totaux de la RAMQ pour les corps étrangers oculaires superficiels. Comme le ratio CÉOS/100 000 des ophtalmologistes n'a augmenté que légèrement, l'augmentation des coûts est plus probablement attribuable à l'augmentation des frais facturés à la RAMQ survenue pendant cette période ${ }^{22}$.

Fait intéressant, les optométristes ont pris en charge $44 \%$ des corps étrangers oculaires superficiels en 2016, ce qui n'a représenté que $13 \%$ des coûts associés à ces actes pour la RAMQ. En revanche, les ophtalmologistes ont pris en charge $21 \%$ des corps étrangers oculaires superficiels, ce qui a représenté $47 \%$ des coûts associés à ces actes pour la RAMQ. Ce résultat pourrait être attribuable au fait qu'en 2016, le coût moyen facturé à la RAMQ pour chaque prise en charge de corps étrangers oculaires superficiels par un optométriste était plus faible (13 \$ CA) (données non présentées) que celui des ophtalmologistes (95 \$ CA) (données non présentées). De plus, comme la RAMQ ne rembourse que la partie diagnostique des consultations visant la prise en charge de corps étrangers oculaires superficiels effectuée par les optométristes, les patients doivent payer la partie thérapeutique (extraction) en consultation privée. En 2016, l'Association des optométristes du Québec a proposé de facturer entre 39 et 57 dollars canadiens pour cet acte ${ }^{23}$. Certains régimes d'assurance privés couvrent ces frais. Les patients qui n'en ont pas peuvent payer de leur poche, ce qui favorise peutêtre un accès plus rapide à un optométriste. Cependant, au bout du compte, lorsque les optométristes prennent en charge un corps étranger oculaire superficiel au Québec, une partie du total des coûts est transférée du système public au patient ou à son assurance privée complémentaire. Ce n'est pas le cas lorsque le corps étranger oculaire superficiel est pris en charge par un ophtalmologiste, un médecin de famille ou un urgentologue.

Cette étude comporte plusieurs limites et pourrait notamment compter des erreurs au sujet des codes de facturation et des frais facturés à la RAMQ. Pour les omnipraticiens et les urgentologues ainsi que pour les ophtalmologistes, il existe de nombreux codes de tarification correspondant à la prise en charge des corps étrangers oculaires superficiels. Or, les codes sont parfois gérés par plusieurs agents de facturation, ce qui augmente le risque d'erreurs. Ce risque est potentiellement moindre en ce qui a trait aux optométristes car ils ne facturent les actes faisant intervenir un corps étranger oculaire superficiel qu'avec un code et un sous-code.

Une autre limite est imposée par la définition d'événement associé à un corps étrangers oculaires superficiels (patient qui a eu au moins une consultation facturée à la RAMQ par année de référence), qui peut occulter le fait qu'une même personne pourrait avoir plus d'un corps étranger oculaire superficiel dans une même année. Une analyse différente aurait pu être réalisée à l'aide des données de la RAMQ afin de produire le nombre d'examens dans la population correspondant à un code de diagnostic des corps étrangers oculaires superficiels. Cela aurait inclus tous les cas, y compris ceux qui ont consulté pour la présence de plusieurs corps étrangers oculaires superficiels au cours de la même année. Néanmoins, comme de nombreux épisodes de corps étrangers oculaires superficiels donnent lieu à des visites de suivi, le risque de surestimer le nombre de cas était plus élevé que dans la méthode d'analyse retenue en raison de l'incapacité de faire la distinction une visite initiale et une visite de suivi pour un même épisode de corps étrangers oculaires superficiels.

Enfin, dans certaines circonstances, les corps étrangers oculaires superficiels peuvent être pris en charge sans que la procédure particulière soit facturée à la RAMQ. Ces cas sont donc exclus de l'analyse en cours. Il peut s'agir, par exemple, de cas pris en charge par des infirmières praticiennes dans des régions éloignées ou par des ophtalmolo- 
gistes ou des résidents en médecine familiale lorsqu'aucun médecin traitant n'est sur place et qu'il est impossible de facturer la RAMQ en se basant sur une procédure, comme cela se produit durant certains quarts de nuit. Cependant, on pense que le nombre de ces cas est faible comparativement au nombre total de cas inclus correspondant aux données présentées ici.

\section{CONCLUSION}

Au Québec, après qu'ils aient vu leur champ d'exercice s'élargir en vertu de la loi, les optométristes sont devenus les professionnels qui prennent en charge la plus grande partie des corps étrangers oculaires superficiels dans la province. Le transfert de ce fardeau semble provenir de la part des corps étrangers oculaires superficiels pris en charge par des omnipraticiens en contexte d'urgence, laquelle a diminué dans une proportion similaire. Cette situation pourrait réduire la pression et les temps d'attente dans les situations d'urgence. Les coûts des soins de santé du régime public (RAMQ) liés aux corps étrangers oculaires superficiels sont plus faibles lorsque les optométristes prennent en charge les corps étrangers oculaires superficiels, mais certains de ces coûts sont transférés hors du système public.

\section{REMERCIEMENTS}

Les auteurs remercient l'Association des optométristes du Québec qui a financé la collecte de données ainsi que la Régie de l'assurance-maladie du Québec, le Collège des médecins du Québec, la Fédération des médecins omnipraticiens du Québec et le service des archives de l'Hôpital général juif de Montréal pour leur collaboration à la collecte et à la mise en contexte des données dans le cadre de cette étude.

\section{DÉCLARATION D'INTÉRÊT}

Aucune

AUTEUR-RESSOURCE

Benoit Tousignant, benoit.tousignant@umontreal.ca

\section{RÉFÉRENCES}

1. Burrillon C. [Foreign bodies in the anterior segment]. J Fr Ophtalmol. 2001;24:751-757.

2. Islam SS, Doyle EJ, Velilla A, Martin CJ, Ducatman AM. Epidemiology of compensable work-related ocular injuries and illnesses: incidence and risk factors. J Occup Environ Med. 2000;42:575-581.

3. Ahmed F, House RJ, Feldman BH. Corneal Abrasions and Corneal Foreign Bodies. Prim Care. 2015;42:363-375.

4. Gonul S, Bozkurt B, Okudan S. Metallic corneal foreign bodies: an occupational health hazard. Arq Bras Oftalmol. 2014;77:411.

5. Gumus K, Karakucuk S, Mirza E. Corneal injury from a metallic foreign body: an occupational hazard. Eye Contact Lens. 2007;33:259260.

6. Aziz MA, Rahman MA. Corneal foreign body--an occupational hazard. Mymensingh Medical Journal : MMJ. 2004;13:174-176.

7. Newell SW. Management of corneal foreign bodies. Am Fam Physician. 1985;31:149-156.

8. Canadian Ophthalmological Society, 2019. http://www.cos-sco.ca/ vision-health-information/your-ophthalmologist/ Accessed: 25-022019

9. Collège des médecins du Québec, 2019. http://www.cmq.org/hub/ $\mathrm{fr} /$ statistiques.aspx Accessed: 2019-02-25

10. Resnikoff S, Felch W, Gauthier TM, Spivey B. The number of ophthalmologists in practice and training worldwide: a growing gap despite more than 200,000 practitioners. Br J Ophthalmol. 2012;96:783-787.

11. Jeannin A, Mouriaux F, Mortemousque B. [Management of ophthalmologic emergencies in general emergency departments: A retrospective multicenter study]. J Fr Ophtalmol. 2016;39:589-595.

12. ESG Média [Index Santé], 2017. http://www.indexsante.ca/urgences/ Accessed: 2017-03-02
13. Gouvernement du Québec. Optometry Act. chapter O-7, s. 19.4, 2018. http://legisquebec.gouv.qc.ca/en/showdoc/cr/O-7,\%20r.\%2011.1. Accessed: 2019-02-25

14. Ordre des optométristes de Québec, 2017. https://www.ooq.org/ collaboration-interprofessionnelle/loptometriste-en-tant-queressource-de-premiere-ligne-en-sante Accessed: 20-03-2017

15. Bellan L. The geographic distribution of optometrists in Ontario. Can J Ophthalmol. 2015;50:92-93.

16. Institut de la Statistique du Québec. Perspectives démographiques du Québec et des régions, 2011-2061. 2014. http://www.stat.gouv. qc.ca/statistiques/population-demographie/perspectives/perspectives-2011-2061.pdf, Accessed 2019-02-25.

17. Institut de la statistique Quebec. Profils statistiques par région et MRC géographiques (Statistical profiles by regions). 2010-2016. http://www.stat.gouv.qc.ca/statistiques/profils/region_00/region_00.htm, Accessed 2019-02-25.

18. Gouvernement du Québec. Act respecting occupational health and safety. chapter S-2.1, 2017. http://legisquebec.gouv.qc.ca/en/ShowDoc/cs/S-2.1. Accessed: 2019-02-25

19. Collège des médecins du Québec, 2016. http://www.cmq.org/ publications-pdf/p-4-2016-06-09-fr-rapport-annuel-2015-2016.pdf Accessed: 2019-02-25

20. ESG Média [Index Santé], 2017. http://www.indexsante.ca/urgences/ Accessed: 2019-02-25

21. Régie de l'assurance maladie du Québec. Manuel des optométristes, no 2402016 .

22. La Régie de l'assurance maladie du Québec. Manuel des médecins spécialistes Montréal: RAMQ; 2017.

23. Association des optométristes du Québec. Guide sommaire des tarifs (Annual fees guide). Montréal, Quebec: 2017. https://www. aoqnet.qc.ca/, Accessed 2019-02-25. 